\title{
TREM2 brain transcript-specific studies in $A D$ and TREM2 mutation carriers
}

Jorge L. Del-Aguila1,2,3, Bruno A. Benitez ${ }^{1,2,3}$, Zeran Li ${ }^{1,2,3}$, Umber Dube ${ }^{1,2,3}$, Kathie A. Mihindukulasuriya $a^{1,2}$, John P. Budde 1,2,3, Fabiana H. G. Farias, 1,2,3, Maria Victoria Fernández ${ }^{1,2,3}$, Laura Ibanez ${ }^{1,2,3}$, Shan Jiang, Richard J. Perrin ${ }^{3,4,5}$, Nigel J. Cairns ${ }^{3,4,5,6}$, John C. Morris ${ }^{4,5}$, Oscar Harari ${ }^{1,2,3,4}$ and Carlos Cruchaga ${ }^{1,2,34^{*}}$ (D)

\begin{abstract}
Background: Low frequency coding variants in TREM2 are associated with Alzheimer disease (AD) risk and cerebrospinal fluid (CSF) TREM2 protein levels are different between AD cases and controls. Similarly, TREM2 risk variant carriers also exhibit differential CSF TREM2 levels. TREM2 has three different alternative transcripts, but most of the functional studies only model the longest transcript. No studies have analyzed TREM2 expression levels or alternative splicing in brains from AD and cognitively normal individuals. We wanted to determine whether there was differential expression of TREM2 in sporadic-AD cases versus ADTREM2 carriers vs sex- and aged-matched normal controls; and if this differential expression was due to a particular TREM2 transcript.
\end{abstract}

Methods: We analyzed RNA-Seq data from parietal lobe brain tissue from AD cases with TREM2 variants $(n=33), A D$ cases $(n=195)$ and healthy controls $(n=118)$, from three independent datasets using Kallisto and the R package tximport to determine the read count for each transcript and quantified transcript abundance as transcripts per million.

Results: The three TREM2 transcripts were expressed in brain cortex in the three datasets. We demonstrate for the first time that the transcript that lacks the transmembrane domain and encodes a soluble form of TREM2 (STREM2) has an expression level around $60 \%$ of the canonical transcript, suggesting that around $25 \%$ of the sTREM 2 protein levels could be explained by this transcript. We did not observe a difference in the overall TREM2 expression level between cases and controls. However, the isoform which lacks the 5' exon, but includes the transmembrane domain, was significantly lower in TREM2- p.R62H carriers than in AD cases ( $p=0.007)$.

Conclusion: Using bulk RNA-Seq data from three different cohorts, we were able to quantify the expression level of the three TREM2 transcripts, demonstrating: (1) all three transcripts of them are highly expressed in the human cortex, (2) that up to $25 \%$ of the sTREM 2 may be due to the expression of a specific isoform and not TREM2 cleavage; and (3) that TREM2 risk variants do not affect expression levels, suggesting that the effect of the TREM2 variants on CSF levels occurs at post-transcriptional level.

Keywords: TREM2, RNAseq, Soluble TREM2, R47H, Alzheimer's disease, Brain transcripts, Risk variants

\footnotetext{
*Correspondence: cruchagac@wustl.edu; ccruchaga@wustl.edu

'Department of Psychiatry, Washington University School of Medicine, St. Louis, MO, USA

${ }^{2}$ NeuroGenomics and Informatics, Washington University School of Medicine,

St. Louis, MO, USA

Full list of author information is available at the end of the article
}

(c) The Author(s). 2019 Open Access This article is distributed under the terms of the Creative Commons Attribution 4.0 International License (http://creativecommons.org/licenses/by/4.0/), which permits unrestricted use, distribution, and reproduction in any medium, provided you give appropriate credit to the original author(s) and the source, provide a link to the Creative Commons license, and indicate if changes were made. The Creative Commons Public Domain Dedication waiver (http://creativecommons.org/publicdomain/zero/1.0/) applies to the data made available in this article, unless otherwise stated. 


\section{Introduction}

The Triggering Receptor Expressed in Myeloid cells 2 (TREM2) is a type 1 transmembrane receptor protein expressed on myeloid cells including microglia, monocyte-derived dendritic cells, osteoclasts and bone-marrow derived macrophages [1, 2]. TREM2 possesses an immunoglobulin-like extracellular domain, a transmembrane region and a short cytoplasmatic tail. In the brain, it is primarily expressed by microglia and has been shown to control two signaling pathways: regulation of phagocytosis and suppression of inflammatory reactivity $[3,4]$. In the case of phagocytosis, there is a very strong relationship between TREM2 and the adaptor protein DAP12, also called TYROBP [5]. Homozygous loss-of-function mutations in TREM2 or DAP12 cause a rare and fatal disease known as Nasu-Hakola disease (NHD) or polycystic lipomembranous osteodysplasia with sclerosing leukoencephalopathy (PLOSL) which is characterized by an early-onset frontotemporal dementia-like phenotype and bone cysts $[6,7]$. One of the leading hypotheses to explain the pathology associated with NHD is that lack of activity of TREM2 or DAP12 causes microglia inactivation and the accumulation of necrotic debris from apoptotic neurons [8].

This relationship with NHD prompted an effort to identify allelic variants in the TREM2 coding region that could also confer risk to Alzheimer's disease. In 2013, several studies found that heterozygous expression of TREM2 p.R47H [9-24] and p.D87N [15] variants were significantly associated with $\mathrm{AD}$ risk. Other variants associated with the risk of $\mathrm{AD}$ include p.D87N $[13,15]$, p.R62H [17, 24], p.L211P, p.T96K and p.H157Y [16, 17]. Among all these variants, p.R47H was validated in neuropathological-confirmed cases [25] and was shown to increase the risk for late onset AD to a similar extent as the ApoE \&4 allele $[15,18]$. However, the functional impact of the variant is not completely understood. In in-vitro studies, p.R47H has been shown to reduce the binding of $\mathrm{A} \beta$ oligomers, APOE and phosphatidylserine due to structural changes in TREM2 [26-29]. In in-vivo studies, the expression of human p.R47H in Trem2 knockout mice suggested that the mutation confers a loss-of-function phenotype [30]. In 2018, two independent studies [31, 32] generated Trem2 R47H knock-in mice using CRISPR/Cas 9 technology. Both mice showed reduced Trem 2 mRNA and protein levels, however the study from Xinag et al. [32] found that TREM2 mRNA level in IPSC-derived human microglia-like cells and in patients' brains with p.R47H were normal.

Most of TREM2 AD-risk variants are located in exon 2, which codes for the Ig-like V type domain, suggesting a possible modification in the interaction between TREM2 and its ligands. Only two other risk variants associated to AD are located in different exons, p.H157Y in exon 3 and p.L211P in exon 4 of the transcript that encodes the soluble form of TREM2 [15]. Kober et al. [33], presents an interesting hypothesis regarding loss of function in TREM2. In NHD, the TREM2 protein is not expressed, or it is expressed in a misfolded form that does not appear in the membrane; either scenario leads to a complete loss of function and a severe early-onset dementia. In $\mathrm{AD}$, the risk variants are expressed in the cell membrane, but their binding capacity is lower than that of the WT, leading to a partial loss of function causing a less severe late-onset dementia. This hypothesis could explain why heterozygous NHD variants, including p.Q33X, p.Y38C, and p.T66 M, have been found in rare AD cases in heterozygous sate [15].

In 2014, it was reported that there are at least three TREM2 transcripts that are expressed in human brain [17]. The first isoform, ENST00000373113, is the canonical and the longest TREM2 transcript which consists of five exons. This transcript has a transmembrane domain, and it is the transcript that is normally modeled in functional studies. The second isoform, ENST00000373122, lacks exon 5, is the second longest transcript, and also includes the transmembrane domain. Both isoforms are anchored to the cell membrane due to their transmembrane domain, and both isoforms can undergo a sequential proteolytic processing by disintegrin and metalloproteinase domain-containing protein (ADAM) family, including ADAM10 and ADAM17, leading to the shedding of the ectodomain and producing a soluble TREM2 $[34,35]$. The third isoform, ENST00000338469, is the shortest with an alternative spliced isoform that excludes exon 4. This isoform most likely encodes a soluble form of TREM2 (sTREM2) due to the lack of exon 4 which encodes the transmembrane domain of the receptor.

A recent study [36] has shown the relevance of cerebrospinal fluid (CSF) STREM2 as a biomarker for AD progression due to its elevation in AD patients. It is unknown whether the sTREM2 is only the cleavage product of the cell-surface expressed protein or also the expression of the shortest soluble form.

The goal of this study is to quantify the expression level of the three TREM2 transcripts in AD and control brains, to determine whether there is differential expression of TREM2 in the three groups, sporadic-AD cases (cases), AD-TREM2 (TREM2-carriers) carriers and controls (controls) and finally to determine whether these differences are due to the differential expression of a particular TREM2 isoform.

\section{Materials and methods}

\section{Subjects and samples}

The number of participants is presented in Table 1; they were grouped into three cohorts: Washington University in St. Louis Knight-ADRC Brain Bank (51 participants), 
Table 1 Demographic Characteristics of study participants

\begin{tabular}{|c|c|c|c|}
\hline & Controls & Alzheimer's Disease Sporadic (Cases) & TREM2-carriers \\
\hline \multicolumn{4}{|l|}{ Knight-ADRC } \\
\hline No. of patients & 12 & 24 & 15 \\
\hline Gender (\% male) & 33.3 & 45.8 & 53.3 \\
\hline Mean age at death (SD), years & $90.1(8.9)$ & $85.1(8.6)$ & $84.5(6.3)$ \\
\hline Mean RIN (SD) & $6.7(1.2)$ & $5.9(1.4))$ & $6.8(1.3)$ \\
\hline APOE genotype (ع4+)\% & 8.3 & 45.8 & 53.3 \\
\hline \multicolumn{4}{|l|}{ Mount Sinai Brain Bank (MSBB-BM36) } \\
\hline No. of patients & 28 & 93 & 11 \\
\hline Gender (\% male) & 50 & 32.3 & 45.5 \\
\hline Mean age at death (SD), years & $80.9(9.1)$ & $84.4(7.2)$ & $84.3(4.6)$ \\
\hline Mean RIN (SD) & $6.6(1.1)$ & $5.9(1.5)$ & $6.0(1.7)$ \\
\hline APOE genotype (ع4+)\% & 11.8 & 60 & 50 \\
\hline \multicolumn{4}{|l|}{ Mayo Clinic Brain Bank (MCBB) } \\
\hline No. of patients & 78 & 77 & 7 \\
\hline Gender (\% male) & 51.3 & 42.9 & 14.3 \\
\hline Mean age at death (SD), years & $82.5(8.9)$ & $82.3(7.8)$ & $81.6(4.6)$ \\
\hline Mean RIN (SD) & $7.6(1.0)$ & $8.6(0.6)$ & $8.3(0.3)$ \\
\hline APOE genotype (ع4+)\% & 10.3 & 51.9 & 57.1 \\
\hline
\end{tabular}

MSBB-BM36, (132 participants) and MCBB (162 participants) all of whom were European-Americans. Briefly, the Knight-ADRC cohort included 15 participants with late-onset $\mathrm{AD}$ (NIA-AA criteria: intermediate or high) [37] with known risk variants in TREM2 gene (Table 2), 12 non-demented controls and 39 sporadic late-onset AD cases. Because the variants p.D87N and p.R136W were associated with NHD and likely lead to neurodegeneration through a different mechanism from that of the AD risk variants, they were not used in the meta-analysis for
TREM2 carriers. For MSBB-BM36, the number of non-demented control and sporadic late-onset $\mathrm{AD}$ cases were 28 and 93, respectively, and a total of 11 AD cases carried a TREM2 risk variant (see Table 2). In the case of MSBB-BM36, there were 78 non-demented controls, 77 sporadic late-onset $\mathrm{AD}$, and $7 \mathrm{AD}$ cases with a TREM2 risk variant (see Table 2). The RNA quality for samples in the study was good, with an average RNA integrity (RIN) number over five in all the cohorts. The age of death was also consistent in all cohorts, with a mean over 80 years old.

Table 2 Number of subjects with the TRME2 variants in each study

\begin{tabular}{llll}
\hline TREM2 Variants & Knight-ADRC & Mount Sinai Brain Bank (MSBB-BM36) & Mayo Clinic Brain Bank (MCBB) \\
\hline p.D87N ${ }^{a}$ & 1 & 0 & 0 \\
p.G219C & 0 & 0 & 0 \\
p.R62C & 0 & 1 & 0 \\
p.E151K & 0 & 0 & 0 \\
p.H157Y & 1 & 0 & 0 \\
p.L211P_T96K & 1 & 0 & 0 \\
p.R136W & 1 & 0 & 0 \\
p.R52H & 1 & 0 & 0 \\
p.L133L & 0 & 1 & 1 \\
p.R47H & 4 & 6 & 1 \\
p.R62H & 8 & 3 & 5 \\
\hline
\end{tabular}

a Variants associated to NHD phenotype, when present on homozigous state

L211P_T96K: These two variants are in LD and are analyzed as a group 


\section{Washington University in St. Louis knight-ADRC brain bank}

Brain tissue was provided by the Washington University in St. Louis Charles F. and Joanne Knight Alzheimer's Disease Research Center Brain Bank (Knight ADRC), all cases were recruited as research participants and underwent a standard battery of tests [38]. Clinical Dementia Rating (CDR) scores were obtained and the estimated CDR at the time of death was determined by telephone interview [39]. Neuropathological assessment was undertaken by NJC and RJP, with each case assessed using the NIA-AA neuropathologic diagnostic criteria. Additional postmortem data including post-mortem interval and brain weight were also available [40].

From these clinically and neuropathologically wellcharacterized cases [41], one to two grams of frozen (-80C) parietal lobe tissue (inferior parietal lobe) was dissected and made available for this study. For each case, post-mortem consent for a brain-only autopsy brain was obtained and was approved by Washington University in St. Louis institutional review board. Briefly, RNA was extracted from frozen brain tissues using the Tissue Lyser LT and RNeasy Mini Kits (Qiagen, Hilden, Germany) following the manufacturer's instructions. RIN and DV200 were measured with the RNA 6000 Pico Assay on the Bioanalyzer 2100 (Agilent Technologies). The RIN is determined by the Bioanalyzer, taking into account the entire electrophoretic trace of the RNA including the presence or absence of degradation products. All the RIN values were acceptable for further analysis. The DV200 value is defined as the \% of nucleotides greater than $200 \mathrm{nt}$. Each sample yield was determined by the Quant-iT RNA Assay (Life Technologies) on the Qubit Fluorometer (Fisher Scientific). The cDNA libraries were prepared with the TruSeq Stranded Total RNA Sample Prep with Ribo-Zero Gold kits (Illumina) and then sequenced by HiSeq 4000 (Illumina) at the McDonnell Genome Institute, Washington University in St. Louis. RNA-seq paired end reads with a read length of $2 \times 150$ bp were generated using Illumina HiSeq 4000 with a mean coverage of 80 million reads per sample.

\section{Mayo Clinic brain Bank (MCBB)}

Mayo Clinic Brain Bank RNA-seq data was downloaded from the AMP-AD portal (synapse ID =5,550,404; accessed January 2017). Paired end reads of $2 \times 101$ base pairs were generated by the Illumina HiSeq 2000 sequencer, for an average of 134.9 million reads per sample. RNA-seq based transcriptome data was generated from post-mortem brain tissue collected from the cerebellum (273 samples) and temporal cortex (275 samples) of Caucasian subjects. For this study, the temporal cortex samples were chosen. RNA was extracted using Trizol $^{\circledR}$ and cleaned with the Qiagen RNeasy kit. RIN measurements were performed with Agilent Technologies 2100 Bioanalyzer. Libraries were prepared by the Mayo Clinic Medical Genome Facility Gene Expression and Sequencing Cores with the TruSeq RNA Sample Prep Kit (Illumina).

\section{Mount Sinai brain Bank (MSBB)}

Mount Sinai Brain Bank RNA-seq data was downloaded from the AMP-AD portal (synapse ID = 3,157,743; accessed January 2017). Single end reads of 100 nucleotides were generated by the Illumina HiSeq 2500 System (Illumina, San Diego, CA), for an average of 38.7 million reads per sample. (Bank, 2016 \#3723). It contains 1030 samples collected from four post-mortem brain regions of 300 subjects: the anterior prefrontal cortex (BM10), the superior temporal gyrus (BM22), parahippocampal gyrus (BM36), and the inferior frontal gyrus (BM44). RNA-seq was generated using the TruSeq RNA Sample Preparation Kit v2 and Ribo-Zero rRNA removal kit (Illumina, San Diego, CA) [https://www.synapse.org/ \#!Synapse:syn3157743]. The parahippocampal gyrus (BM36) is a cortical region in the medial temporal lobe that projects to surrounds the hippocampus and plays an important role in both spatial memory [42] and navigation $[43,44]$. For this reason, BM36 was selected for further analysis for this study.

\section{TREM2 variant calling}

For Knight ADRC, TREM2 was sequenced using pooled-DNA sequencing designed as described previously [45]. All polymorphisms were validated by Sequenom and KASPar genotype for each individual included in the pool. For Mayo and the Mount Sinai datasets, TREM2 variant calling was performed separately for $30 \mathrm{X}$ WGS as described before [46-48], following GATK's 3.6 Best Practices (https://www.broadinstitute.org/gatk/) Variants were called in a region defined by the capture targets of the Agilent SureSelect Human All Exon V5 Kit, plus $100 \mathrm{bp}$ of padding added to each capture target end. WGS data was filtered to remove low complexity regions, and regions with excessive depth. Only those variants that scored above the $99.5 \%$ confidence threshold were considered for analysis; additional variant filters included allele-balance $(\mathrm{AB}=0.3-0.7)$. We also performed variant calling in the RNA-seq to determine if the TREM2 variants were expressed. We confirm that in all cases the alternative allele is expressed validating the genotypes for all individuals.

\section{RNA-seq expression quantification}

FastQC [49] was used to assess sequencing quality. The RNA-seq was aligned to the human GRCh37 primary assembly using STAR (ver 2.5.2b). Read alignments were further evaluated by using PICARD CollectRnaSeqMetrics 
(ver 2.8.2) to examine read distribution across the genome. We employed Kallisto (v0.42.5) [50] and tximport [51] to determine the read count for each transcript and quantified transcript abundance as transcripts per kilobase per million reads mapped (TPM), using gene annotation of Homo sapiens reference genome (GENCODE GRCh37) for each participant from Knight-ADRC, MCB and MSBB-BM36 independently, with the following parameters: - $t 10$-b 100. Then we summed the read counts and TPM of all alternative splicing transcripts of a gene to obtain gene expression levels. Due to the positive skewness of TPM values, we calculate their logarithm 10 $(\log 10 \mathrm{TPM})$ for further analysis.

\section{Immunoprecipitation}

Immunoprecipitation was performed as previously described [52]. In brief, brain tissue were lysed in T-PER buffer (Thermo Scientific) and 1X Protease Inhibitor Cocktail (Sigma-Aldrich). Brain homogenate were precleared for $1 \mathrm{~h}$ at $4{ }^{\circ} \mathrm{C}$ with $20 \mathrm{uL}$ of Pierce ${ }^{\mathrm{met}}$ Protein G Agarose (Thermo Scientific). TREM2 protein was immunoprecipitated overnight at $4{ }^{\circ} \mathrm{C}$ with $5 \mu \mathrm{g}$ of purified mouse monoclonal anti-TREM2 antibodies directed against the extra-cellular portion of human TREM2 protein (clone 20G2 and 29E3). $20 \mu \mathrm{L}$ of Protein G agarose were added to the antigen-antibody complex and incubated for $2 \mathrm{~h}$ at room temperature. Precipitates were washed four times in cold phosphate buffer saline (PBS) with protease inhibitors (Sigma-Aldrich). SDS gel-loading buffer was added to the complex-bound resin, incubated for $5 \mathrm{~min}$ at $95^{\circ} \mathrm{C}$, separated by SDS-PAGE, transferred to PVDF membranes (BIO-RAD) and probed with the mouse anti-TREM-2 mAbs supernatants (clones 10B11 and 21E10). Densitometric semi-quantification was performed using ImageJ software (National Institutes of Health) [53].

\section{Statistical analysis}

We employed a linear regression model to test the association between the $\log 10$ TPM and each comparison group (R Foundation for Statistical Computing, ver.3.3.3) for the Knight-ADRC, MCBB and MSBB (BM36) cohorts. RIN number, age at death and gender were used as covariates for each analysis. All studies showed different absolute $\log 10$ TPM values, due to the different library preparation or brain region. Therefore, for the meta-analysis, we used Stouffer's Z-score method, which is based on Z-scores rather than $p$-values, allowing incorporation of study weights based on the sample size for each cohort. The definition of statistical significance was nominal $P$-value $(p<0.05)$ and the same direction of effects (that is, the sign of $\beta$ ) in each study to avoid discounting true positive associations.

\section{Results}

All three TREM2 transcripts are highly expressed in human cortex

We first wanted to determine whether TREM2 transcripts are expressed in the brain cortex, then to quantify each transcript to determine their relative abundance and possible importance. In order to be able to combine and compare expression levels across datasets, we normalized the $\log 10 \mathrm{TPM}$ using the mean expression of the longest transcript as a reference. Log10TPM counts and analyses results can be found in the Additional file (Additional file 1: Table S1). We found that the canonical TREM2 (ENST00000373113) transcript has significantly higher expression than the other two transcripts $\left(p=1.13 \times 10^{-120}\right.$ and $4.76 \times 10^{-107}$, respectively, Fig. 1, Tables 3 and 4). The transcript that encodes the soluble TREM2 isoform (ENST00000338469) had the second highest expression level, and it is expressed at $62 \%$ of the canonical transcript. The transcript with the lowest expression level is the transcript that encodes a shorted transmembrane protein (ENST00000373122) and is expressed at 58\% of the level of the canonical transcript. This transcript also showed a significantly lower level of expression than the transcript that encodes the soluble form $(p=0.0003)$.

We also performed similar analyses for stratified by case-control status, and for each dataset, separately. The expression levels were very consistent across datasets and independent of case-control status (Fig. 1, Tables 3 and 4). The relative expression of the transcript that encodes the soluble form (ENST00000338469) ranges from 53 to $65 \%$ of that of the canonical transcript (ENST00000373113) and for the ENST00000373122 transcript, expression ranges from 55 to $61 \%$. This data confirms that the three isoforms are expressed and that does not correlate with case-control status, and that up to $25 \%$ of the extracellular sTREM 2 could be due to the expression of the ENST00000338469 isoform and not a product of TREM2 cleavage.

\section{Correlation with protein levels}

In order to determine if the transcript levels correlate with protein levels, we quantified TREM2 protein levels by Western Blot on 10 parietal brain samples from the Knight-ADRC that RNA-seq data was also available. We detected two bands (Additional file 1: Figure S1); the $50 \mathrm{kD}$ band correspond to the full length TREM2 [34]. The second band located $\sim 30 \mathrm{kD}$, which matches to the sTREM2 found in cerebrospinal fluid [52]. Then, we quantified the band corresponding to the sTREM2 band and analyzed if there was a correlation with the sTREM2 band with the different transcripts. We found a very strong correlation between the sTREM2 band vs the total mRNA TREM2 levels $\left(R^{2}=0.77 p<0.05\right)$. We also found that the 


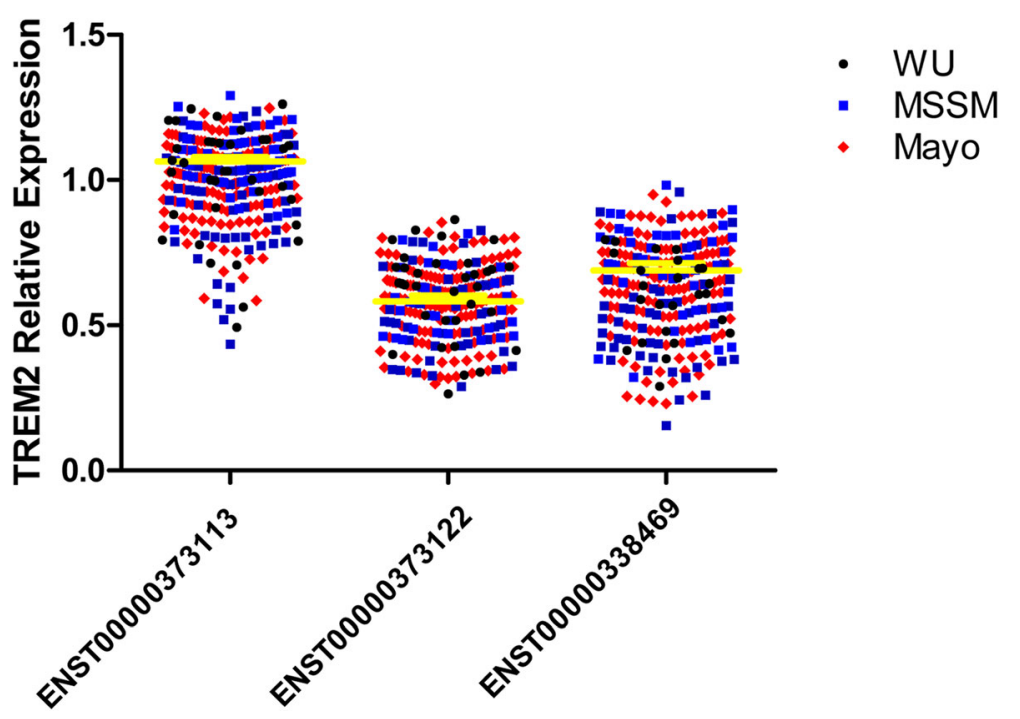

Fig. 1 Relative quantification of the expression of each isoform in Alzheimer's disease cases, controls and TREM2 risk variant carriers. Total log10 RNA count was calculated using Kallisto and the results were expressed relative to the log10RNA counts of the common transcript ENST00000373113. There is a clear overlapping distribution for each of the studies, the yellow line represents the mean overall value for each transcript among the different studies. There were different expression counts among the different transcripts (see Table 5)

STREM2 band shown a correlation with the canonical isoform $\left(R^{2}=0.73 \quad p<0.05\right)$ and the isoform that codify for the soluble transcript $\left(\mathrm{R}^{2}=0.42 p<0.05\right)$, but not for the other transcript $\left(\mathrm{R}^{2}=-0.13 p>0.05\right)$.

TREM2 expression levels are not affected by disease status

Since CSF sTREM2 levels are significantly different in $\mathrm{AD}$ cases vs controls, our next step was to determine whether the overall TREM2 gene expression and its transcript specific expression were associated with case-control status. For these analyses, TREM2 carriers were not included. The overall TREM2 expression levels were higher in $\mathrm{AD}$ cases compared to controls, but this difference was not statistically significant $(p=0.11$, Table 5). Similar results were found for the canonical transcript. However, we found a nominal association between the transcript that encodes STREM2, in which

Table 3 Relative quantification of the expression of each isoform with ENST00000373113 as control

\begin{tabular}{|c|c|c|c|c|}
\hline Study & Conditions & ENST00000373113 (canonical transcript) & ENST00000373122 & ENST00000338469 (soluble TREM2) \\
\hline \multirow[t]{3}{*}{ All } & Control + Case & 1.00 & 0.58 & 0.62 \\
\hline & Control & 0.97 & 0.56 & 0.62 \\
\hline & Case & 1.02 & 0.58 & 0.63 \\
\hline \multirow[t]{3}{*}{ Knight-ADRC } & Control + Case & 1.00 & 0.61 & 0.60 \\
\hline & Control & 0.97 & 0.56 & 0.53 \\
\hline & Case & 1.01 & 0.64 & 0.63 \\
\hline \multirow[t]{3}{*}{ MSBB-BM36 } & Control + Case & 1.00 & 0.56 & 0.61 \\
\hline & Control & 0.94 & 0.55 & 0.60 \\
\hline & Case & 1.02 & 0.56 & 0.61 \\
\hline \multirow[t]{3}{*}{ MCBB } & Control + Case & 1.00 & 0.58 & 0.64 \\
\hline & Control & 0.98 & 0.57 & 0.63 \\
\hline & Case & 1.02 & 0.60 & 0.64 \\
\hline
\end{tabular}

ENST00000373113 canonical transcript 
Table 4 Wilcoxon test for unpaired two independent groups

\begin{tabular}{|c|c|c|c|c|}
\hline Study & Conditions & $\begin{array}{l}\text { ENST00000373113 vs } \\
\text { ENST00000373122 }\end{array}$ & $\begin{array}{l}\text { ENST00000373113 vs } \\
\text { ENST00000338469 }\end{array}$ & $\begin{array}{l}\text { ENST00000373122 vs } \\
\text { ENST00000338469 }\end{array}$ \\
\hline \multirow[t]{3}{*}{ All } & $\begin{array}{l}\text { Control + } \\
\text { Case }\end{array}$ & $1.13 \times 10^{-120}$ & $4.76 \times 10^{-107}$ & 0.0002 \\
\hline & Control & $1.57 \times 10^{-43}$ & $2.61 \times 10^{-35}$ & 0.0090 \\
\hline & Case & $1.86 \times 10^{-79}$ & $2.80 \times 10^{-74}$ & 0.0095 \\
\hline \multirow[t]{3}{*}{$\begin{array}{l}\text { Knight- } \\
\text { ADRC }\end{array}$} & $\begin{array}{l}\text { Control + } \\
\text { Case }\end{array}$ & $2.16 \times 10^{-12}$ & $3.39 \times 10^{-11}$ & 0.6423 \\
\hline & Control & $6.66 \times 10^{-05}$ & 0.0003 & 0.4789 \\
\hline & Case & $1.07 \times 10^{-07}$ & $1.58 \times 10^{-07}$ & 0.7850 \\
\hline \multirow[t]{3}{*}{ MSBB-BM36 } & $\begin{array}{l}\text { Control + } \\
\text { Case }\end{array}$ & $2.99 \times 10 \mathrm{e}^{-40}$ & $1.95 \times 10^{-38}$ & 0.0291 \\
\hline & Control & $6.20 \times 10^{-08}$ & $3.86 \times 10^{-07}$ & 0.3969 \\
\hline & Case & $1.86 \times 10^{-34}$ & $2.79 \times 10^{-33}$ & 0.0475 \\
\hline \multirow[t]{3}{*}{ MCBB } & $\begin{array}{l}\text { Control + } \\
\text { Case }\end{array}$ & $1.99 \times 10^{-72}$ & $2.16 \times 10^{-61}$ & 0.0003 \\
\hline & Control & $1.04 \times 10^{-33}$ & $3.43 \times 10^{-26}$ & 0.0051 \\
\hline & Case & $9.33 \times 10^{-43}$ & $9.98 \times 10^{-39}$ & 0.0238 \\
\hline
\end{tabular}

Table shows the $p$-value for the test comparison among studies and each isoforms:

ENST00000373113 canonical transcript

ENST00000373122

ENST00000338469 soluble TREM2

AD cases have higher transcript levels $(p=0.04$, Table 5$)$. These results were consistent across datasets replicating this finding (Additional file 1: Tables S2-S4).

TREM2 risk variants do not affect TREM2 expression levels or disease status

We also decided to analyze whether the TREM2 risk variants (mainly p.R47H and p.R62H) were associated with TREM2 expression levels. We also included some variants that in homozygosity cause NHD, although in this study all the individuals with NHD variants are in a heterozygous state. NHD variants are loss-of-function variants that lead to very low TREM2 cell surface expression and low sTREM2 levels [36]. AD risk variants have also been postulated to be partial loss-of-function. Although, it is known that the loss-of-function for the NHD variants requires a post-transcriptional event, we wanted to confirm that these NHD or AD-risk variants have no effect at the mRNA level. We found that NHD variant-carriers have similar TREM2 expression levels to $\mathrm{AD}$ cases, controls and other TREM2 variants (Fig. 2). We also did not see any specific TREM2 transcript effect for the NHD variants. All the transcripts were expressed at similar levels than in non-mutation carriers.

Then, we determined if the overall TREM2 or specific transcript levels were significantly different between TREM2 variant carriers (any TREM2 variant) and cases or controls. We did not find any significant association,

Table 5 TREM2 is similarly expressed in AD, controls and TREM2 risk variant carriers

\begin{tabular}{|c|c|c|c|c|}
\hline & Overall TREM2 & ENST00000373113 (canonical transcript) & ENST00000373122 & ENST00000338469 (soluble TREM2) \\
\hline Control vs Case & 0.11 & 0.2 & 0.19 & $0.04^{c}$ \\
\hline Control vs TREM2-carriers & 0.40 & 0.39 & 0.31 & 0.39 \\
\hline Case vsTREM2-carriers & 0.11 & 0.26 & 0.19 & 0.15 \\
\hline Control vs p.R47H & 0.33 & 0.34 & 0.15 & 0.06 \\
\hline Case vs p.R47H ${ }^{a}$ & 0.24 & 0.38 & 0.35 & 0.11 \\
\hline Control vs p.R62H & 0.41 & 0.48 & $0.06^{\mathrm{b}}$ & 0.25 \\
\hline Case vs p.R62H & 0.17 & 0.20 & $0.007^{b}$ & 0.22 \\
\hline
\end{tabular}

Table shows the p-value for the meta-analysis for Knight-ADRC, Mayo Clinic Brain Bank and Mount Sinai Brain Bank

Meta-analyses was performed using the Zscore Method

Statistical significant results with the same effect size direction are shown in red

${ }^{a}$ Data only available for Knight-ADRC and Mount Sinai Brain Bank

${ }^{b}$ Data only available for Knight-ADRC and Mayo Clinic Brain Bank

'Statistically significant results but with different effect size directions 


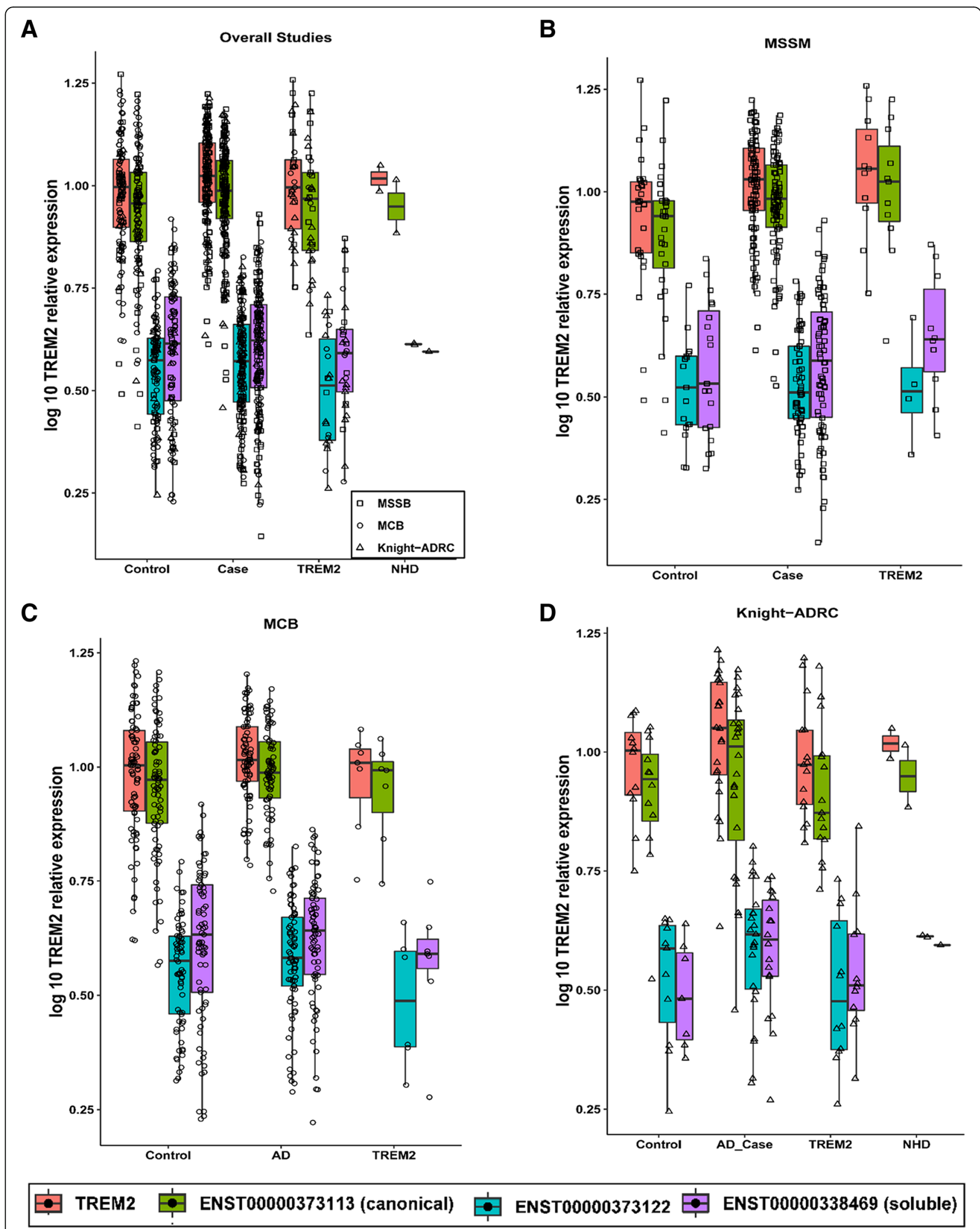

Fig. 2 Overall TREM2 and transcript-specific expression in Alzheimer's disease cases, controls and TREM2 risk variant carriers. Gene expression is showed as a log10 TPM values. a Overall, (b) Mount Sinai Brain Bank (MSBB) -BM36 study, (c) Mayo Clinic Brain Bank study and (d) Knight-ADRC study. Overall TREM2 refers to the total TREM2 expression of all the three transcripts 
although TREM2-carriers showed nominally significant lower levels of the ENST00000373122 transcript (shorter, transmembrane protein) compared to controls (Fig. 2 and Table 5).

Finally, we performed specific analyses for the p.R47H and p.R62H variants. We did not find any significant difference in the overall or transcript-specific TREM2 levels for the p.R47H variant. On the other hand, we found that the p.R62H variant carriers have significantly lower expression of the second transmembrane TREM2 transcript (ENST00000373122) than cases $(p=0.007)$, and have lower expression compared to controls, although this difference is not statistically significant (Fig. 2 and Table 5). We did not find any difference for the other two transcripts or for the overall expression, suggesting that this effect is transcript specific.

\section{Discussion}

Various studies suggest that TREM2 and its variants contribute to the pathogenesis of AD [54], Parkinson's Disease [55], and Amyotrophic Lateral Sclerosis risk [56]. However, it remains unclear if TREM2 is a pro- or anti-inflammatory molecule [57]. For instance, in studies in macrophages or microglia, TREM2 expression reduces the inflammatory response $[4,58-60]$, while in dendritic cells, TREM2 expression exacerbates the inflammatory response [2, 61]. Due to these apparently conflicting results, a hypothesis was developed in which TREM2 function depends on which cell type it is expressed. Therefore, if the cells are part of the innate immune system, TREM2 acts as an anti-inflammatory molecule, but if they are part of the adaptive immune system, TREM2 acts as a pro-inflammatory molecule. Most of the current research has been focused on TREM2 gene expression levels and the TREM2 variants associated with $\mathrm{AD}$ risk, however most of the studies have only focused on the canonical transcript, and not the activity or expression levels of the TREM2 isoforms. It is also important to determine the expression levels of the three TREM2 transcripts, as it is important to distinguish between the expression of the soluble TREM2 isoform (ENST00000338469) and the s TREM2 produced by proteolytic cleavage. It is known that sTREM2 plays a role in AD pathogenesis [54] and can be used as biomarker for $\mathrm{AD}$. Interestingly, the results from these studies shown that TREM2 levels are higher in AD individuals in CSF samples [36], which are consistent with our results at mRNA levels. However, one of the three TREM2 transcripts expresses a soluble form of TREM2 that could be also be released extracellularly and be part of the overall pool of sTREM2 levels. Therefore, if we are to understand the role of sTREM2 in disease, we need to determine all the potential origins of this protein.
The expression of the three TREM2 transcripts in brain was initially reported by Jin et.al [17]. In other studies, the expression of the canonical transcript (ENST00000373113) was the highest [62], followed by the transcript which encodes soluble TREM2 (ENST00000338469) [63]. In this study, we used a large cohorts, totaling 345 samples to analyze not only the expression levels of the three TREM2 transcripts but also to determine the association of $\mathrm{AD}$ case-control status and the TREM2 risk variants with the expression levels.

We were able to detect and quantify the levels of three TREM2 transcripts ENST00000373113, ENST000003 73122 and ENST00000338469 using RNA-seq data from $\mathrm{AD}$ and control brains from three different, independent studies. Our analyses indicate that the canonical transcript (ENST00000373113) is expressed at twice the level of the other two other transcripts, and that this difference is very consistent across studies and between cases and controls. Even with this, our results indicate that the transcript that encodes STREM2 represents the $25 \%$ of the total TREM2 mRNA suggesting that around $20-25 \%$ of the sTREM2 might be due to the expression of this transcript and not the cleavage of the cell membrane bound TREM2. In order to try to determine how much of the sTREM2 is produced by each transcript, we quantify total and sTREM2 from brain homogenates using Western-blots. As expected, we found a strong correlation of the STREM2 band with the total TREM2 mRNA $\left(R^{2}=0.77 p<0.05\right)$ and the canonical transcript $\left(\mathrm{R}^{2}=0.73 p<0.05\right)$. But we also found a strong and significant correlation with the transcript that codify for the soluble TREM2 transcript $\left(\mathrm{R}^{2}=0.42 p<0.05\right)$. This data supports that a proportion of the sTREM2 could be the results of the expression of the transcript that codify for the soluble isoform. However, there are several limitations to this experiment. Antibody-based detection assays are not able to distinguish between sTREM2 that was generated by proteolytic processing [35] or by alternative splicing [17]. Most of the epitopes used to generate anti-TREM2 antibodies are located in the extracellular portion of human TREM2 protein, which is shared by both the proteolytic and the transcribed forms $[34,35,52,64]$. Therefore we are not able to determine the transcript of origin of the sTREM2 protein. The second limitation is that, because we quantified sTREM2 from brain homogenates, we can not distinguish between the extra and intra-cellular sTREM 2 proteins. Therefore we cannot demonstrate that the sTREM2 protein produced by the soluble transcript is also present extracellularly. In addition, the TREM2 in the membrane is not all cleaved immediately. The actual proportion of sTREM2 that is produced by the direct expression of the ENST00000338469 transcript could be significantly higher or lower than $25 \%$. More refined and specialized 
experimental techniques are needed to determine the origin of the soluble and extracellular presence of sTREM2.

In any case, recent studies indicate that minor changes in sTREM2 levels, of around $7-10 \%$ are enough to modulate $\mathrm{AD}$ risk [54, 65]. Therefore changes of expression levels by $25 \%$ should have a large impact on AD risk. Additional research to understand transcript specific TREM2 regulation and determine the origin of sTREM2 are needed to fully understand the biology of TREM2.

Two previous papers [31, 32], that generated Trem 2 R47H knock-in mice using CRISPR/Cas 9 technology, showed reduced Trem 2 mRNA and protein levels, although the same studies suggest that these results were an artifact due to some unspecific events linked to the genome editing. It is also known that NHD variants are loss-of-function mutations. For instance, p.Y38C disrupts the correct disulfide formation, p.T66 M destabilizes the protein expression and p.V126G disrupts hydrophobic core of the protein. Furthermore, p.Y38 and p.V126 are conserved within the TREM family [33], implying that they are probably required to preserve the common fold within this family of receptors leading to lower sTREM2 levels, due to post-transcriptional events. On the other hand, variants p.R47H, p.R62H, p.N68K, p.D87N and p.T96K lie on the protein surface and are related to $\mathrm{AD}$ risk. Based on the location of these variants, we assumed that they would not affect surface expression and instead impact ligand binding [33]. Several studies indicate that CSF TREM2 levels are increased in $\mathrm{AD}$ risk variant p.R47H carriers, but in this case it is not clear if this is post-transcriptional, at the mRNA level. In our meta-analysis, the overall expression of TREM2 at the gene level, and transcript-specific analyses in p.R47H or NHD carriers, TREM2 and its isoforms were not significantly differentially expressed when compared to controls or AD cases, suggesting that the effects associated with the p.R $47 \mathrm{H}$ variant are also at the post-transcriptional level. On the other hand, our data indicate the p.R62H carriers showed a significant level of the ENST0000037312, which also encodes a transmembrane TREM2 isoform. This finding could explain why TREM2 p.R62H carriers tend to have lower CSF TREM2 levels [36, 54].

\section{Conclusion}

This study demonstrates that the three TREM2 transcripts are highly expressed in human brains, and that the transcript that encodes the soluble form of TREM2 might represent up to $25 \%$ of the soluble TREM2. Therefore, our results indicate that this transcript may play an important role in disease, and additional studies are needed to functionally characterize the rol of this transcript in disease. The implication of this transcript in $\mathrm{AD}$ pathogenesis is also supported by the presence of $\mathrm{AD}$ risk variant that are only present in the transcript that codifies for the soluble form. [17]. Thus in order to understand the biology of TREM2, it is important to perform functional studies and create cell and animal models that interrogate the three transcripts, not only the canonical transcript.

In this study, we were able to demonstrate the expression of the three transcripts of TREM2 in postmortem human brain, including the soluble transcript that does not include a transmembrane domain. We also found the AD-risk variants influenced the expression of specific transcripts.

\section{Additional file}

Additional file 1: Table S1. TREM2 mean expression levels for each transcript and in each study. Table S2. Knight-ADRC results from each comparison group. Table S3. Mount Sinai Brain Bank (MSBB)-BM36 results from each comparison group. Table S4 Mayo Clinic Brain Bank (MCBB) results from each comparison group. Figure S1. Immuno precipitation sTREM2 in brain samples (DOCX $199 \mathrm{~kb}$ )

\section{Abbreviations}

AD: Alzheimer's disease; ADAM: A disintegrin and metalloproteinase domaincontaining protein; BM10: Anterior prefrontal cortex; BM22: Superior temporal gyrus; BM36: Parahippocampal gyrus; BM44: Inferior frontal gyrus; CDR: Clinical Dementia Rating; CSF: Cerebrospinal fluid; DAP12: DNAX activation protein of $12 \mathrm{kDa}$; Knight-ADRC: Washington University in St. Louis Charles F. and Joanne Knight Alzheimer's Disease Research Center Brain Bank; log10TPM: Logarithm10 TPM; MCBB: Mayo Clinic Brain Bank; MSBB: Mount Sinai Brain Bank; NHD: Nasu-Hakola Disease; PLOSL: Polycystic Lipomembranous Osteodysplasia with Sclerosing Leukoencephalopathy; STREM2: Soluble form of TREM2; TPM: Transcripts per Million reads mapped; TREM2: Triggering Receptor Expressed on Myeloid cells 2; TYROBP: TYRO protein tyrosine kinase binding protein

\section{Acknowledgments}

We thank all the participants and their families, as well as the many institutions and their staff that provided support for the studies involved in this collaboration.

\section{Funding}

This work was supported by grants from the National Institutes of Health (R01AG044546, P01AG003991, RF1AG053303, R01AG058501, U01AG058922, RF1AG058501 and R01AG057777), the Alzheimer Association (NIRG-11-200110, BAND-14-338165, AARG-16-441560 and BFG-15-362540). BAB is supported by 2018 pilot funding from the Hope Center for Neurological Disorders and the Danforth Foundation Challenge at Washington University. The recruitment and clinical characterization of research participants at Washington University were supported by NIH P50 AG05681, P01 AG03991, and P01 AG026276. This work was supported by access to equipment made possible by the Hope Center for Neurological Disorders, and the Departments of Neurology and Psychiatry at Washington University School of Medicine.

\section{Availability of data and materials}

1. Data for the Knight-ADRC is available to qualified investigators by applying to the Knight ADRC website https://knightadrc.wustl.edu/Research/ResourceRequest.htm.

2. Mayo RNAseq Study data were provided by the following sources: The Mayo Clinic Alzheimer's Disease Genetic Studies, led by Dr. Nilufer Taner and Dr. Steven G. Younkin, Mayo Clinic, Jacksonville, FL using samples from the Mayo Clinic Study of Aging, the Mayo Clinic Alzheimer's Disease Research Center, and the Mayo Clinic Brain Bank. 
Data collection was supported through funding by NIA grants P50 AG016574, R01 AG032990, U01 AG046139, R01 AG018023, U01 AG006576, U01 AG006786, R01 AG025711, R01 AG017216, R01 AG003949, NINDS grant R01 NS080820, CurePSP Foundation, and support from Mayo Foundation. Study data includes samples collected through the Sun Health Research Institute Brain and Body Donation Program of Sun City, Arizona. The Brain and Body Donation Program is supported by the National Institute of Neurological Disorders and Stroke (U24 NS072026 National Brain and Tissue Resource for Parkinson's Disease and Related Disorders), the National Institute on Aging (P30 AG19610 Arizona Alzheimer's Disease Core Center), the Arizona Department of Health Services (contract 211002, Arizona Alzheimer's Research Center), the Arizona Biomedical Research Commission (contracts 4001, 0011, 05-901 and 1001 to the Arizona Parkinson's Disease Consortium) and the Michael J. Fox Foundation for Parkinson's Research.

3. MSBB: These data were generated from postmortem brain tissue collected through the Mount Sinai VA Medical Center Brain Bank and were provided by Dr. Eric Schadt from Mount Sinai School of Medicine.

\section{Authors' contributions}

JLDA analyzed the RNAseq data, performed bioinformatics analyses, and wrote the manuscript. FF, ZL, UD, KM and OH performed the geneexpression analyses in human brain tissue. ZL, UD, MVF, LI, SJ and JB performed the QC for the all data. RP, NC, JM, OH, CC supervised and wrote the project. All authors read and approved the manuscript.

\section{Ethics approval and consent to participate}

The protocol of Knight-ADRC studies have been approved by the review board of Washington University in St. Louis. The protocol of Mayo dataset was approved by the Mayo Clinic Institutional Review Board (IRB). All neuropsychological, diagnostic and autopsy protocols of MSSM dataset were approved by the Mount Sinai and JJ Peters VA Medical Center Institutional Review Boards. The MSSM sample protocol was approved by Icahn School of Medicine at Mount Sinai IRB; Al participants were recruited with informed consent for research use.

\section{Consent for publication}

Not applicable

\section{Competing interests}

CC receives research support from: Biogen, EISAl, Alector and Parabon. The funders of the study had no role in the collection, analysis, or interpretation of data; in the writing of the report; or in the decision to submit the paper for publication. CC is a member of the advisory board of ADx Healthcare, and Vivid Genomics.

\section{Publisher's Note}

Springer Nature remains neutral with regard to jurisdictional claims in published maps and institutional affiliations.

\section{Author details}

'Department of Psychiatry, Washington University School of Medicine, St. Louis, MO, USA. ${ }^{2}$ NeuroGenomics and Informatics, Washington University School of Medicine, St. Louis, MO, USA. ${ }^{3}$ Hope Center for Neurological Disorders, Washington University School of Medicine, St. Louis, MO, USA. ${ }^{4}$ Knight Alzheimer's Disease Research Center, Washington University School of Medicine, St. Louis, MO, USA. ${ }^{5}$ Department of Neurology, Washington University School of Medicine, St. Louis, MO, USA. ${ }^{6}$ Department of Pathology and Immunology, Washington University School of Medicine, St. Louis, MO, USA

Received: 20 November 2018 Accepted: 26 April 2019

Published online: 08 May 2019

\section{References}

1. Bouchon A, Dietrich J, Colonna M. Cutting edge: inflammatory responses can be triggered by TREM-1, a novel receptor expressed on neutrophils and monocytes. J Immunol. 2000;164:4991-5.
2. Bouchon A, Hernandez-Munain C, Cella M, Colonna M. A DAP12-mediated pathway regulates expression of CC chemokine receptor 7 and maturation of human dendritic cells. J Exp Med. 2001;194:1111-22.

3. Neumann $\mathrm{H}$, Takahashi K. Essential role of the microglial triggering receptor expressed on myeloid cells-2 (TREM2) for central nervous tissue immune homeostasis. J Neuroimmunol. 2007:184:92-9.

4. Takahashi K, Prinz M, Stagi M, Chechneva O, Neumann H. TREM2-transduced myeloid precursors mediate nervous tissue debris clearance and facilitate recovery in an animal model of multiple sclerosis. PLoS Med. 2007;4:e124.

5. Klesney-Tait J, Turnbull IR, Colonna M. The TREM receptor family and signal integration. Nat Immunol. 2006;7:1266-73.

6. Klunemann $\mathrm{HH}$, Ridha BH, Magy L, Wherrett JR, Hemelsoet DM, Keen RW, De Bleecker JL, Rossor MN, Marienhagen J, Klein HE, et al. The genetic causes of basal ganglia calcification, dementia, and bone cysts: DAP12 and TREM2. Neurology. 2005;64:1502-7.

7. Paloneva J, Manninen T, Christman G, Hovanes K, Mandelin J, Adolfsson R, Bianchin M, Bird T, Miranda R, Salmaggi A, et al. Mutations in two genes encoding different subunits of a receptor signaling complex result in an identical disease phenotype. Am J Hum Genet. 2002;71:656-62.

8. Thrash JC, Torbett BE, Carson MJ. Developmental regulation of TREM2 and DAP12 expression in the murine CNS: implications for Nasu-Hakola disease. Neurochem Res. 2009;34:38-45.

9. Benitez BA, Cooper B, Pastor P, Jin SC, Lorenzo E, Cervantes S, Cruchaga C TREM2 is associated with the risk of Alzheimer's disease in Spanish population. Neurobiol Aging. 2013;34:1711 e1715-7.

10. Benitez BA, Jin SC, Guerreiro R, Graham R, Lord J, Harold D, Sims R, Lambert JC, Gibbs JR, Bras J, et al. Missense variant in TREML2 protects against Alzheimer's disease. Neurobiol Aging. 2014;35:1510 e1519-26.

11. Cuyvers E, Bettens K, Philtjens S, Van Langenhove T, Gijselinck I, van der Zee J, Engelborghs S, Vandenbulcke M, Van Dongen J, Geerts N, et al. Investigating the role of rare heterozygous TREM2 variants in Alzheimer's disease and frontotemporal dementia. Neurobiol Aging. 2014;35:726 e711-29.

12. Finelli D, Rollinson S, Harris J, Jones M, Richardson A, Gerhard A, Snowden J, Mann D, Pickering-Brown S. TREM2 analysis and increased risk of Alzheimer's disease. Neurobiol Aging. 2015;36:546 e549-13.

13. Ghani M, Sato C, Kakhki EG, Gibbs JR, Traynor B, St George-Hyslop P, Rogaeva E. Mutation analysis of the MS4A and TREM gene clusters in a case-control Alzheimer's disease data set. Neurobiol Aging. 2016;42(217): e217-217 e213.

14. Giraldo M, Lopera F, Siniard AL, Corneveaux JJ, Schrauwen I, Carvajal J, Munoz C, Ramirez-Restrepo M, Gaiteri C, Myers AJ, et al. Variants in triggering receptor expressed on myeloid cells 2 are associated with both behavioral variant frontotemporal lobar degeneration and Alzheimer's disease. Neurobiol Aging. 2013;34:2077 e2011-78.

15. Guerreiro R, Wojtas A, Bras J, Carrasquillo M, Rogaeva E, Majounie E, Cruchaga C, Sassi C, Kauwe JS, Younkin S, et al. TREM2 variants in Alzheimer's disease. N Engl J Med. 2013;368:117-27.

16. Jiang T, Tan L, Chen Q, Tan MS, Zhou JS, Zhu XC, Lu H, Wang HF, Zhang YD, Yu JT. A rare coding variant in TREM2 increases risk for Alzheimer's disease in Han Chinese. Neurobiol Aging. 2016;42:217 e211-3.

17. Jin SC, Benitez BA, Karch CM, Cooper B, Skorupa T, Carrell D, Norton JB, Hsu S. Harari O, Cai Y, et al. Coding variants in TREM2 increase risk for Alzheimer's disease. Hum Mol Genet. 2014;23:5838-46.

18. Jonsson T, Stefansson H, Steinberg S, Jonsdottir I, Jonsson PV, Snaedal J, Bjornsson S, Huttenlocher J, Levey Al, Lah JJ, et al. Variant of TREM2 associated with the risk of Alzheimer's disease. N Engl J Med. 2013;368: $107-16$.

19. Lill CM, Rengmark A, Pihlstrom L, Fogh I, Shatunov A, Sleiman PM, Wang LS, Liu T, Lassen CF, Meissner E, et al. The role of TREM2 R47H as a risk factor for Alzheimer's disease, frontotemporal lobar degeneration, amyotrophic lateral sclerosis, and Parkinson's disease. Alzheimer's Dement. 2015;11:1407-16.

20. Mehrjoo Z, Najmabadi A, Abedini SS, Mohseni M, Kamali K, Najmabadi H, Khorram Khorshid HR. Association study of the TREM2 gene and Identification of a novel variant in exon 2 in Iranian patients with late-onset Alzheimer's disease. Med Princ Pract. 2015;24:351-4.

21. Rosenthal SL, Bamne MN, Wang X, Berman S, Snitz BE, Klunk WE, Sweet RA, Demirci FY, Lopez OL, Kamboh MI. More evidence for association of a rare TREM2 mutation (R47H) with Alzheimer's disease risk. Neurobiol Aging. 2015;36:2443 e2421-46. 
22. Ruiz A, Dols-Icardo O, Bullido MJ, Pastor P, Rodriguez-Rodriguez E, Lopez de Munain A, de Pancorbo MM, Perez-Tur J, Alvarez V, Antonell A, et al. Assessing the role of the TREM2 p.R47H variant as a risk factor for Alzheimer's disease and frontotemporal dementia. Neurobiol Aging. 2014;35:444 e441-4.

23. Slattery CF, Beck JA, Harper L, Adamson G, Abdi Z, Uphill J, Campbell T, Druyeh R, Mahoney CJ, Rohrer JD, et al. R47H TREM2 variant increases risk of typical early-onset Alzheimer's disease but not of prion or frontotemporal dementia. Alzheimer's Dement. 2014;10:602-608 e604.

24. Yu JT, Jiang T, Wang YL, Wang HF, Zhang W, Hu N, Tan L, Sun L, Tan MS, Zhu XC, Tan L. Triggering receptor expressed on myeloid cells 2 variant is rare in late-onset Alzheimer's disease in Han Chinese individuals. Neurobiol Aging. 2014;35:937 e931-3

25. Roussos P, Katsel P, Fam P, Tan W, Purohit DP, Haroutunian V. The triggering receptor expressed on myeloid cells 2 (TREM2) is associated with enhanced inflammation, neuropathological lesions and increased risk for Alzheimer's dementia. Alzheimer's Dement. 2015;11:1163-70.

26. Sudom A, Talreja S, Danao J, Bragg E, Kegel R, Min X, Richardson J, Zhang Z, Sharkov N, Marcora E, et al. Molecular basis for the loss-of-function effects of the Alzheimer's disease-associated $\mathrm{R} 47 \mathrm{H}$ variant of the immune receptor TREM2. J Biol Chem. 2018;293:12634-46.

27. Wang Y, Cella M, Mallinson K, Ulrich JD, Young KL, Robinette ML, Gilfillan S, Krishnan GM, Sudhakar S, Zinselmeyer BH, et al. TREM2 lipid sensing sustains the microglial response in an Alzheimer's disease model. Cell. 2015; 160:1061-71.

28. Yeh FL, Wang Y, Tom I, Gonzalez LC, Sheng M. TREM2 binds to apolipoproteins, including APOE and CLU/APOJ, and thereby facilitates uptake of amyloid-Beta by microglia. Neuron. 2016;91:328-40.

29. Zhao Y, Wu X, Li X, Jiang LL, Gui X, Liu Y, Sun Y, Zhu B, Pina-Crespo JC, Zhang $M$, et al. TREM2 is a receptor for beta-amyloid that mediates microglial function. Neuron. 2018;97:1023-31 e1027.

30. Song WM, Joshita S, Zhou Y, Ulland TK, Gilfillan S, Colonna M. Humanized TREM2 mice reveal microglia-intrinsic and -extrinsic effects of R47H polymorphism. J Exp Med. 2018;215:745-60.

31. Cheng-Hathaway PJ, Reed-Geaghan EG, Jay TR, Casali BT, Bemiller SM, Puntambekar SS, von Saucken VE, Williams RY, Karlo JC, Moutinho M, et al. The Trem2 R47H variant confers loss-of-function-like phenotypes in Alzheimer's disease. Mol Neurodegener. 2018;13:29.

32. Xiang X, Piers TM, Wefers B, Zhu K, Mallach A, Brunner B, Kleinberger G, Song W, Colonna M, Herms J, et al. The Trem2 R47H Alzheimer's risk variant impairs splicing and reduces Trem2 mRNA and protein in mice but not in humans. Mol Neurodegener. 2018;13:49.

33. Kober DL, Alexander-Brett JM, Karch CM, Cruchaga C, Colonna M, Holtzman MJ, Brett TJ. Neurodegenerative disease mutations in TREM2 reveal a functional surface and distinct loss-of-function mechanisms. eLife. 2016;5: e20391. https://doi.org/10.7554/eLife.20391.

34. Kleinberger $G$, Yamanishi $Y$, Suarez-Calvet M, Czirr E, Lohmann $E$, Cuyvers $E$, Struyfs H, Pettkus N, Wenninger-Weinzierl A, Mazaheri F, et al. TREM2 mutations implicated in neurodegeneration impair cell surface transport and phagocytosis. Sci Transl Med. 2014;6:243ra286.

35. Wunderlich P, Glebov K, Kemmerling N, Tien NT, Neumann H, Walter J. Sequential proteolytic processing of the triggering receptor expressed on myeloid cells-2 (TREM2) protein by ectodomain shedding and gammasecretase-dependent intramembranous cleavage. J Biol Chem. 2013;288: 33027-36.

36. Piccio L, Deming Y, Del-Aguila JL, Ghezzi L, Holtzman DM, Fagan AM, Fenoglio C, Galimberti D, Borroni B, Cruchaga C. Cerebrospinal fluid soluble TREM2 is higher in Alzheimer disease and associated with mutation status. Acta Neuropathol. 2016;131:925-33.

37. Montine TJ, Phelps CH, Beach TG, Bigio EH, Cairns NJ, Dickson DW, Duyckaerts C, Frosch MP, Masliah E, Mirra SS, et al. National Institute on Aging-Alzheimer's Association guidelines for the neuropathologic assessment of Alzheimer's disease: a practical approach. Acta Neuropathol. 2012;123:1-11.

38. Mirra SS, Heyman A, McKeel D, Sumi SM, Crain BJ, Brownlee LM, Vogel FS, Hughes JP, van Belle G, Berg L. The consortium to establish a registry for Alzheimer's disease (CERAD). Part II. Standardization of the neuropathologic assessment of Alzheimer's disease. Neurology. 1991;41:479-86.

39. Morris JC. Clinical dementia rating: a reliable and valid diagnostic and staging measure for dementia of the Alzheimer type. Int Psychogeriatr. 1997;9(Suppl 1):173-6; discussion 177-178.
40. Gavett BE, John SE, Gurnani AS, Bussell CA, Saurman JL. The role of Alzheimer's and cerebrovascular pathology in mediating the effects of age, race, and apolipoprotein E genotype on dementia severity in pathologically-confirmed Alzheimer's disease. J Alzheimer's Dis. 2016;49: 531-45.

41. Li Z, Del-Aguila JL, Dube U, Budde J, Martinez R, Black K, Xiao Q, Cairns NJ, Dominantly Inherited Alzheimer N, Dougherty JD, et al. Genetic variants associated with Alzheimer's disease confer different cerebral cortex cell-type population structure. Genome Med. 2018;10:43.

42. Squire LR, Zola-Morgan S. The medial temporal lobe memory system. Science. 1991;253:1380-6.

43. Aguirre GK, Detre JA, Alsop DC, D'Esposito M. The parahippocampus subserves topographical learning in man. Cereb Cortex. 1996;6:823-9.

44. Maguire EA, Burke T, Phillips J, Staunton H. Topographical disorientation following unilateral temporal lobe lesions in humans. Neuropsychologia. 1996;34:993-1001

45. Del-Aguila JL, Fernandez MV, Schindler S, Ibanez L, Deming Y, Ma S, Saef B, Black K, Budde J, Norton J, et al. Assessment of the genetic architecture of Alzheimer's disease risk in rate of memory decline. J Alzheimer's Dis. 2018; 62:745-56.

46. Fernandez MV, Black K, Carrell D, Saef B, Budde J, Deming Y, Howells B, DelAguila JL, Ma S, Bi C, et al. SORL1 variants across Alzheimer's disease European American cohorts. Eur J Hum Genet. 2016;24:1828-30.

47. Fernandez MV, Budde J, Del-Aguila JL, Ibanez L, Deming Y, Harari O, Norton J, Morris JC, Goate AM, Group N-Lfs, et al. Evaluation of gene-based familybased methods to detect novel genes associated with familial late onset Alzheimer disease. Front Neurosci. 2018;12:209.

48. Fernandez MV, Kim JH, Budde JP, Black K, Medvedeva A, Saef B, Deming Y, Del-Aguila J, Ibanez L, Dube U, et al. Analysis of neurodegenerative Mendelian genes in clinically diagnosed Alzheimer disease. PLoS Genet. 2017;13:e1007045

49. FastQC A Quality Control tool for high throughput sequence data [http:// www.bioinformatics.babraham.ac.uk/projects/fastqc/ ].

50. Bray NL, Pimentel H, Melsted P, Pachter L. Near-optimal probabilistic RNAseq quantification. Nat Biotechnol. 2016;34:525-7.

51. Soneson C, Love MI, Robinson MD. Differential analyses for RNA-seq: transcript-level estimates improve gene-level inferences. F1000Research. 2015;4:1521.

52. Piccio L, Buonsanti C, Cella M, Tassi I, Schmidt RE, Fenoglio C, Rinker J 2nd, Naismith RT, Panina-Bordignon P, Passini N, et al. Identification of soluble TREM-2 in the cerebrospinal fluid and its association with multiple sclerosis and CNS inflammation. Brain. 2008;131:3081-91.

53. Benitez BA, Sands MS. Primary fibroblasts from CSPalpha mutation carriers recapitulate hallmarks of the adult onset neuronal ceroid lipofuscinosis. Sci Rep. 2017:7:6332.

54. Deming Y, Li Z, Benitez BA, Cruchaga C. Triggering receptor expressed on myeloid cells 2 (TREM2): a potential therapeutic target for Alzheimer disease? Expert Opin Ther Targets. 2018;22:587-98.

55. Benitez BA, Cruchaga C. United States-Spain Parkinson's disease research G: TREM2 and neurodegenerative disease. N Engl J Med. 2013;369:1567-8.

56. Cady J, Koval ED, Benitez BA, Zaidman C, Jockel-Balsarotti J, Allred P, Baloh RH, Ravits J, Simpson E, Appel SH, et al. TREM2 variant p.R47H as a risk factor for sporadic amyotrophic lateral sclerosis. JAMA Neurol. 2014;71:449-53.

57. Konishi H, Kiyama H. Microglial TREM2/DAP12 signaling: a double-edged sword in neural diseases. Front Cell Neurosci. 2018;12:206.

58. Hamerman JA, Jarjoura JR, Humphrey MB, Nakamura MC, Seaman WE, Lanier LL. Cutting edge: inhibition of TLR and FCR responses in macrophages by triggering receptor expressed on myeloid cells (TREM)-2 and DAP12. J Immunol. 2006:177:2051-5.

59. Piccio L, Buonsanti C, Mariani M, Cella M, Gilfillan S, Cross AH, Colonna M, Panina-Bordignon P. Blockade of TREM-2 exacerbates experimental autoimmune encephalomyelitis. Eur J Immunol. 2007:37:1290-301.

60. Turnbull IR, Gilfillan S, Cella M, Aoshi T, Miller M, Piccio L, Hernandez M, Colonna M. Cutting edge: TREM-2 attenuates macrophage activation. J Immunol. 2006;177:3520-4.

61. Jiang H, Si Y, Li Z, Huang $X$, Chen S, Zheng Y, Xu G, Chen X, Chen Y, Liu Y, et al. TREM-2 promotes acquired cholesteatoma-induced bone destruction by modulating TLR4 signaling pathway and osteoclasts activation. Sci Rep. 2016;6:38761.

62. Celarain N, Sanchez-Ruiz de Gordoa J, Zelaya MV, Roldan M, Larumbe R, Pulido L, Echavarri C, Mendioroz M. TREM2 upregulation correlates with 5- 
hydroxymethycytosine enrichment in Alzheimer's disease hippocampus. Clin Epigenetics. 2016;8:37.

63. Ma L, Allen M, Sakae N, Ertekin-Taner N, Graff-Radford NR, Dickson DW, Younkin SG, Sevlever D. Expression and processing analyses of wild type and p.R47H TREM2 variant in Alzheimer's disease brains. Mol Neurodegener. 2016;11:72.

64. Thornton P, Sevalle J, Deery MJ, Fraser G, Zhou Y, Stahl S, Franssen EH, Dodd RB, Qamar S, Gomez Perez-Nievas B, et al. TREM2 shedding by cleavage at the H157-S158 bond is accelerated for the Alzheimer's diseaseassociated H157Y variant. EMBO Mol Med. 2017;9:1366-78.

65. Deming Y, Filipello F, Cignarella F, Hsu S, Mikesell R, Li Z, Del-Aguila JL, Dube U, Farias FG, Bradley J, Cantoni C, et al. The MS4A gene cluster is a key regulator of soluble TREM2 and Alzheimer disease risk. Sci Transl Med. 2019. manuscript \#aau2291.

\section{Ready to submit your research? Choose BMC and benefit from:}

- fast, convenient online submission

- thorough peer review by experienced researchers in your field

- rapid publication on acceptance

- support for research data, including large and complex data types

- gold Open Access which fosters wider collaboration and increased citations

- maximum visibility for your research: over $100 \mathrm{M}$ website views per year

At $\mathrm{BMC}$, research is always in progress.

Learn more biomedcentral.com/submissions 